\title{
8. POSITIONAL ASTRONOMY (ASTRONOMIE DE POSITION)
}

President: A. A. Nemiro.

Vice-President: W. Fricke.

Organizing Committee: A. N. Adams, P. Lacroute, R. H. Stoy, R. H. Tucker, J. E. B. von der Heide, H. Yasuda, M. S. Zverev.

It is with deep regret that we mention the death, on 15 July 1969, of Prof. Dr G. Demetrescu who has done so much for the development of Astronomy in Roumania.

This report is based on letters received from members of the Commission, whom I wish to thank most cordially.

The most important events in the development of positional astronomy during the three past years were:

(1) The construction and use of new types of astronomical instruments based on new techniques, and

(2) The collective observations of large numbers of stars, especially in the Southern hemisphere on the basis of international cooperation.

\section{THE CONSTRUCTION OF NEW INSTRUMENTS}

At the USNO a design study for a ten-inch automatic meridian circle (ATC) was completed in 1968. Construction and installation should be completed by the end of 1970 . A modified Cassegrainian optical system with a Cer-Vit primary mirror is being used. For some other important parts of the instrument new materials will also be used. At each end of the horizontal axis of the instrument Inductosyns will be mounted.

An Inductosyn system for digital readout of the pointing angle of the telescope was installed on the six-inch transit circle of the USNO in 1967 for testing and calibration. An IBM-1800 data acquisition and control system was on line with the same instrument.

At the Pulkovo Observatory Suharev's mirror transit circle was tested with photoelectrical observations in right ascension of bright FK 4 stars and faint stars of KSZ up to the magnitude 8.5. From April 1967 more than 2500 observations have been made. The results of these observations are promising. The mean error of one determination of $n$ is $\pm_{-}^{3} .010$. The mean pointing error with photoelectrical micrometers equals \pm 0 ."06. The stability of the optical axis of the tubes is good; the variations of their azimuths are less than 0,003 per degree of temperature. The investigation of the instrument and the observations with it are in progress.

The new axis of the mirror transit circle of the Porto Observatory has been installed. The strong dependence of the flexure upon the reaction of the pivots was removed.

Star observations in right ascension have started. Studies are being made for digitizing the circle readings.

On the Canadian mirror circle Dr E. G. Woolsey writes:

"The Ottawa mirror transit project has been abandoned owing to economy measures. The principal difficulties were:

1. A thermal instability of the piers supporting the central section evidenced by a large nightly drift in azimuth.

2. Poor seeing within the long horizontal optical paths, which have been only partially controlled.

To overcome these defects would require expenditure of time and resources which are not available. The instrument and building are on a temporary site and must be dismantled."

The transit circle of the Hamburg Observatory was equipped with a photoelectrical device for observations in both coordinates. The instrument has been installed in Perth (Western Australia). 
Regular observations of the SRS and BSstars and the planets have been started intensively from the end of 1967. The Tokyo meridian circle has been equipped with a new digital micrometer during August 1969. It makes it possible to record observational data in computed readable form. All 2160 diameter corrections were determined by the Bruns-Fayet method with an accuracy of \pm 0.05 (m.e.)

An automatic editing and "marking off" system is being developed at the Royal Greenwich Observatory (Herstmonceux), so that the progress of an observing programme can be monitored by a computer.

The first results Obtained with the tracking photoelectric micrometer of the meridian circle of the Bordeaux Observatory have been confirmed: the m.e. of a single observation is \pm 0.012 for equatorial stars as faint as 9 . 0 . This error is independent of magnitude and spectral type.

Since May 1968 the photoelectric micrometer has been in use on the meridian circle of the Liège Observatory at the experimental station of Dorres (near Font-Romeu, Eastern Pyrenees). Unfortunately, an instability in the azimuth of the meridian circle prevented one obtaining the accuracy expected in this mountain site (height $+1677 \mathrm{~m}$ ).

At the Bordeaux Observatory a new tracking photoelectric micrometer for the simultaneous determination of right ascension and declination is under development.

The micrometer of the meridian circle of the Besançon Observatory is under reconstruction. Photocomputing techniques with a Høg's grating will be introduced.

A new measuring-machine for the declination film is constructed at the Lund observatory. It is hoped to carry out a total determination of the division errors of the new circle in the immediate future.

At the Golosseyevo Observatory a similar photoelectric semi-automatic machine was built. The construction of a new type device for photographing the vertical circle divisions is nearing completion.

An automatic photoelectric machine for measuring the films with circle divisions of the meridian circle of the Odessa Observatory was built.

An analogous machine but of different construction was built at Pulkovo for measuring the films of circle divisions of the meridian circle of the Santiago Observatory (Chile). This machine is already used for reductions of SRS and BS star observations made in Chile in previous years.

A special machine for measuring star tracks on the plates of the Pulkovo photographic vertical circle was also built. It is in the testing phase.

At the Observatory of the Dresden Technical University an astrometric application of the TVtechnique was developed and partly realized. The first results with the new circumzenithal (90, $1350 \mathrm{~mm}$ ) telescope are promising. A new type of pendulum level with electronic equipment was built at the same observatory. The mean error of one level-reading is about \pm 0.06 to \pm 0.09 .

A proposal has been made by Dr D. V. Thomas (Royal Greenwich Observatory) for a reffecting astrolabe, using the aluminized surfaces of a Cer-Vit prism to ensure a stable altitude of observation. An experimental modification of the Danjon astrolabe is envisaged after its return from the Cape.

\section{INTERNATIONAL REFERENCE STAR PROGRAMME}

The President of the AGK3R and SRS Working Groups Dr F. P. Scott reports:

\section{A. $A G K 3 R$ programme}

In accordance with Resolution 5 adopted by Commission 8 at the Berkeley Meeting, all individual observations of AGK3R stars and associated fundamental stars collected at the U.S. Naval Observatory during the programme were sent to the Pulkovo Observatory.

Copies of the catalogue of final positions of 21499 AGK3 stars in machine readable form were sent to the Bergedorf, Pulkovo and Strasbourg Observatories. Copies of this catalogue on magnetic tape may now be obtained on a tape exchange basis. Requests should be addressed to the U.S. Naval Observatory, Washington, D.C. 20390, U.S.A. 
It is hoped that proper motions for the majority of the AGK3R stars may be derived in the not too distant future. A pilot proper motion programme by T. E. Corbin, U. S. Naval Observatory, led to proper motions with mean errors of $\pm 0.35 /$ century for 2679 AGK3R stars with favorable observational histories in the region $+0^{\circ}$ to $+30^{\circ}$. The principal difficulty at the moment is the lack of a sufficient number of earlier catalogues in machine readable form.

About the state of AGK 3 as of December 1, 1969 Prof. W. Dieckvoss and Dr.'H. Kox report:

"Least squares solutions are finished for the 1939 pairs of plates with reference positions from AGK 2 A (reduced to FK 4) and the final AGK 3 R using proper motions from AGK 3 preliminary for reducing to individual epochs of plates. The zones from the pole down to $+36^{\circ}$ declination of AGK 2 (revised) and AGK 3 are on magnetic tapes and on punched cards. Duplicates of the tapes have been sent to the U.S. Naval Observatory Washington and to the Royal Greenwich Observatory. The remaining zones are in preparation. Remeasuring of stars found to be of inferior quality during the first calculations as well as editing magnitudes, spectral types and BD-numbers as auxiliary data is in progress.

Data from individual plates, zones from the pole down to $+35^{\circ}$ so far, were sent to $\mathrm{P}$. Lacroute of Strasbourg, France. Prof. Lacroute uses these data for extensive calculations by modern overlap-methods, including terms depending on magnitude, and applying individual weights to different plates."

Harley Wood of Sydney, Australia, is continuing his photographic work of covering the whole southern hemisphere by a modern wide angle objective aiming at a fourfold overlap. Four zones of $5^{\circ}$ width are finished, three zones are in progress.

Dr. Dorrit Hofffeit of Yale University Observatory reported progress on the Yale Zone Catalog work.

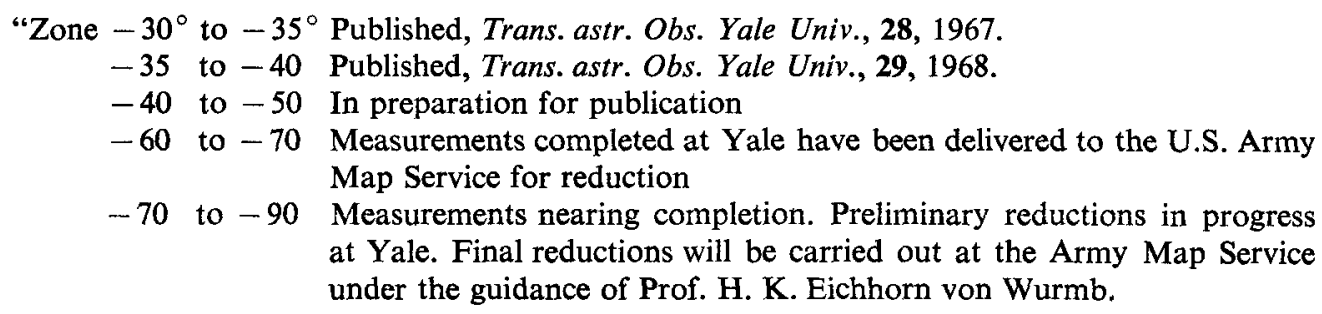

For the future we are contemplating partially remeasuring the plates on the $-40^{\circ}$ to $-50^{\circ}$ zone and adding all of the stars in the Cape Catalogue of Faint Stars which were not included in the Zone Catalogue now being prepared for printing. In addition we plan to measure all possible stars in both of the Cape Catalogues of this zone together with selected galaxies on the first epoch plates that are being taken at the Yale-Columbia Southern Station at El Leoncito. (The older Yale plates, ca. 1940, do not show many galaxies.)"

\section{B. SRS programme}

Dr. F.P. Scott writes: "Table 1 shows the state of the SRS Programme on 1 July 1969.

It is a pleasure to note that the Felix Aguilar Observatory of the National University of Cuyo, San Juan, Argentina commenced its commitment of 2 observations of each of 7190 stars in the zone $-40^{\circ}$ to $-90^{\circ}$ during June 1969 .

The Abbadia commitment has been completed and the results of observation were sent to the U.S. Naval Observatory during October 1968. Approximately $70 \%$ of the Bordeaux results arrived at the U.S. Naval Observatory during July 1967. The remainder is expected during the early months of 1970 . The results for the Cape zone $-40^{\circ}$ to $-52^{\circ}$ were published in the Third Cape Catalogue for $1950 \cdot 0$.

The U.S. Naval Observatory seven-inch transit circle went into operation at El Leoncito, Argentina during May 1967. A few months were spent in testing the performance of the instrument and 
Table 1. State of SRS Programme - 1 July 1969

\begin{tabular}{|c|c|c|c|c|c|c|}
\hline \multirow{2}{*}{ Observatory } & \multirow{2}{*}{ Zone } & \multicolumn{2}{|c|}{ Commitment } & \multirow{2}{*}{$\begin{array}{l}\text { Date } \\
\text { Started }\end{array}$} & \multicolumn{2}{|c|}{ Completed } \\
\hline & & Stars & Obs'ns & & Obs'ns & Red'ns \\
\hline Abbadia & $+5^{\circ}$ to $-15^{\circ}$ & 1560 & 4 & $62 \cdot 3$ & $100 \%$ & $100 \%$ \\
\hline Bordeaux & +5 to -15 & 1560 & 4 & $62 \cdot 5$ & 89 & 100 \\
\hline Bucharest & +5 to -10 & 1176 & 4 & $62 \cdot 6$ & 100 & 45 \\
\hline Nicolaiev & 0 to -20 & 5984 & 2 & $64 \cdot 3$ & 100 & 90 \\
\hline San Fernando & -10 to -30 & 3709 & 4 & $63 \cdot 3$ & 80 & 83 \\
\hline Tokyo & -10 to -30 & 3560 & 4 & $63 \cdot 3$ & 100 & 80 \\
\hline USNO 6-in & +5 to -30 & $\left\{\begin{array}{l}8706 \\
1233\end{array}\right.$ & $\left.\begin{array}{l}2 \\
4\end{array}\right\}$ & $66 \cdot 5$ & 60 & 60 \\
\hline Cape & $\left\{\begin{array}{l}-30 \text { to }-40 \\
-40 \text { to }-50 \\
-50 \text { to }-90\end{array}\right\}$ & 10082 & 4 & $61 \cdot 3$ & $\left\{\begin{array}{r}90 \\
100 \\
0\end{array}\right.$ & $\left.\begin{array}{r}90 \\
100 \\
0\end{array}\right\}$ \\
\hline Santiago-Pulkovo & $\left\{\begin{array}{ll}-25 & \text { to }-47 \\
-47 & \text { to }-90\end{array}\right\}$ & 11496 & 4 & $63 \cdot 1$ & $\left\{\begin{array}{r}90 \\
100\end{array}\right.$ & $\left.\begin{array}{l}35 \\
50\end{array}\right\}$ \\
\hline Bergedorf (Bickley) & +5 to -90 & 20495 & 4 & 67.9 & 43 & 70 \\
\hline $\begin{array}{l}\text { USNO 7-in } \\
\text { (E1 Leoncito) }\end{array}$ & $\begin{cases}+5 & \text { to }-20 \\
-20 & \text { to }-75 \\
-75 & \text { to }-75 S P\end{cases}$ & $\left.\begin{array}{r}7683 \\
12121 \\
1382\end{array}\right\}$ & $\left.\begin{array}{l}2 \\
4 \\
4\end{array}\right\}$ & $68 \cdot 7$ & 25 & 70 \\
\hline San Juan & -40 to -90 & 7190 & 2 & 69.5 & 2 & 2 \\
\hline
\end{tabular}

in training observers before undertaking work on the SRS Programme. In addition to differential zone observations of the reference stars and fundamental stars, special all-night tours of observations are taken about once a week in which all FK 4 and FK 4 Supp stars between $+30^{\circ}$ and $-70^{\circ}$ S.P. are included. The latter series of observations are intended for improvement of the fundamental system. The programme at El Leoncito also includes the Mount Stromlo and La Plata PZT star list, 156 stars in the Scorpio-Centaurus association, all close double stars whose quota of observations had not been completed while the instrument was in Washington, and the four brightest minor planets."

Prof. Slaucitajs reports:

"The meridian circle loaned to La Plata by the Lick Observatory has not been installed in La Plata as yet. This instrument is intended to make the observations corresponding to the SRS Programme. Modernization of the instrument (change of pivots and supports, and overhauling of the eye-piece micrometer) is being done by Carl Zeiss Oberkochen, who have promised to finish the work towards the end of next November. In the meantime the existing pavilion will be reconditioned (slit enlargement) and the rebuilding and modernization of other parts of the instrument will be finished here at the Observatory's shops. The above-mentioned observations with this meridiancircle will start in 1970."

Dr. F. P. Scott concludes his report with the words:

"It is difficult at this time to predict the date of completion of the SRS Programme. However, from the information supplied by the observers, all commitments should be completed by 1973 .

The U.S. Naval Observatory has made over 196000 apparent place, 119000 refraction, and $103000 m, n$, and $c$ computations for the SRS Programme."

Simultaneously with the SRS Programme five major and four bright minor planets were observed at Bickley with the meridian circle of the Bergedorf Observatory. The visual magnitudes of all stars were determined with the photoelectrical device.

With the SRS Programme are connected the works on the photographic catalogues.

S. V. M. Clube reported from the Cape Observatory: 
"Photographic Survey of the Southern Hemisphere

This report describes progress as of September 1969 on the programme outlined briefly in Highlights of Astronomy IAU (1967).

\section{Observations with the Cape camera}

Coverage of the zone $0^{\circ}>\delta>-52^{\circ}$ is virtually complete (4000 plates) and that of $-52^{\circ}>\delta>-90^{\circ}$ well advanced. Arrangements have been made to obtain a series of sub-polar overlapping plates in order to examine the local refraction constant.

\section{Measurements}

All stars in the SRS programme have been measured on the Cape Zone plates (approximately 700 plates in the region $-40^{\circ}>\delta>-52^{\circ}$ ), in order to test analytical procedures.

\section{Analysis}

A reduction procedure using the Herstmonceux ICL 1909 has been developed for these measures, and has so far revealed a radially symmetric distortion pattern, partly dependent on magnitude, which is produced by the objective, and a differential equation between the photographic and meridian positions, mostly in right ascension, of unexplained origin. Eliminating these systematic effects, relative positions of individual stars on the overlapped photographic system are found to be good to at least 0 "05 (s.e.). The material used by Hough in producing the Cape Zone Catalogue for 1900 (Gill and Hough, HMSO 1923) has now been punched ( $\approx 30000$ cards) at Herstmonceux, and is being analysed by the procedure used on the modern plates in order to test the production of a photographic proper motion system. Relative proper motions promise to be as good as 0.002 p.a. (s.e.) over large areas of the sky."

The sixth and final volume of the Cape Photographic Catalogue for 1950 has been published in 1968 (Cape Ann., vol. XXII). It covers the zone between $-80^{\circ}$ and $-90^{\circ}$ for an epoch which is approximately 1954.

Dr. K. Aa. Strand reports from the U.S. Naval Observatory:

"Work on an astrographic catalogue is under way at the U.S. Naval Observatory. The catalogue will cover the sky south of declination $+5^{\circ}$ and will be nearly complete to visual magnitude 9 . Blue and yellow plates will be taken with a new twin astrograph with 2 meter focal length, $f / 10$ cameras (scale: $100^{\prime \prime}$ arc $/ \mathrm{mm}$ ). An automatic engine will be used to measure positions and approximate $B$ and $V$ magnitudes. Both the astrograph and the measuring engine are currently under construction. Dr J. F. Wanner is in charge of this programme."

\section{BS programme}

The following observatories are observing the Bright Stars in their SRS zones: Abbadia, Bordeaux, Bucharest, Nicolahev, Tokyo, Cape, Bergedorf (Bickley) and Santiago-Pulkovo. In addition, Bucharest is observing 586 Bright Stars in the zone $+5^{\circ}$ to $+20^{\circ}$ and the USNO 6-in. all Bright Stars from $-30^{\circ}$ to $+90^{\circ}$. The state of completion of the observations of the Bright Stars at each observatory is approximately the same as it is for the Southern Reference Stars. Observations of Bright Stars in the zone $-10^{\circ}+90^{\circ}$ have been finished at Kiev University Observatory. At the Tashkent Observatory the Bright Stars in the declination zone $-20^{\circ}$ to $+90^{\circ}$ were observed in right ascension only. Nine zone catalogues have been published and one is in press. The Bright Stars in the zone $-15^{\circ}$ to $+90^{\circ}$ are also being observed (right ascensions only) at the Kharkov University Observatory. Thus, as a result of a generous response to a Resolution adopted by Commission 8 at the Moscow Meeting, the Bright Star list is being well observed at this epoch.

\section{DS programme}

The list of double stars which might be troublesome to measure on photographic plates (Draft 
Report Commission 8' Prague Meeting) is being observed as a whole from $-25^{\circ}$ to $-90^{\circ}$ by the Russian and Chilean observers at Santiago. Bucarest is observing 680 double stars, of which 360 are from the above list, in the zone $0^{\circ}$ to $+20^{\circ}$.

Unless other observatories have these stars under observation, there is great danger that they may not be adequately observed at this epoch.

The Double Star List may still be obtained from the U.S. Naval Observatory.

\section{E. $K S Z$ programme}

At Odessa the meridian circle is being used for differential observations in the FK 4 system of 645 FKSZ stars in the zone $-30^{\circ}$ to $+90^{\circ}$. The catalogue will be published at the end of 1970 . Before these observations the instrument was tested and partially modernized (photographing of the $\delta$ micrometer readings, motor-driven travelling thread). The catalogues of KSZ stars (declinations only) in the FK 4 system have been compiled at this observatory ( 973 stars in the zone $-10^{\circ}$ to $-5^{\circ}$ and 422 stars in the zone $-9^{\circ}$ to $+3^{\circ}$ of declination); the catalogues will be published in 1970 .

At the Bucharest Observatory 61 selected KSZ stars surrounding galaxies were observed simultaneously with the observations of the BS and DS stars. About $90 \%$ of the observations are accomplished. The Bucharest $F K S Z$ catalogue ( 643 stars in the zone $-30^{\circ}$ to $90^{\circ}$ ) published in 1957 was revised and improved in the FK 4 system. The results obtained by $M$. Tudor and E. Toma were published in Studii Cerc. Astr., 13, no. 1, 1968. The catalogue of $3913 \mathrm{KSZ}$ stars in the zone $-10^{\circ}$ to $+10^{\circ}$ was also improved in the FK 4 system. The manuscript is in preparation for print.

At the Engelhardt Observatory the same improvement was made for the catalogue of $537 \mathrm{KSZ}$ stars, observed at this observatory. The main results will be published in Trudy 18 astrometr. conf. SSSR."

In Moscow (Sternberg Institute) a catalogue of KSZ stars in the zone $+30^{\circ}$ to $+45^{\circ}$ was published by A. P. Guliaev, Y. A. Korobova and M. P. Homiakova (Trudy gos. astr. Inst. Sternberga v. XXXIX). A catalogue of KSZ stars in the zone $+60^{\circ}$ to $+90^{\circ}$ is in press.

The Moscow catalogues of FKSZ stars 1941 and 1956 were improved and reduced to the FK 4 system by L. M. Hommik using an original method worked out by the author (Trudy gos. astr. Inst. Sternberga, v. XXXIX, Soob̌̌č. gos. astr. Inst. P. K. Sternberga, no. 156).

Observations of the FKSZ stars made in 1948-54 with the meridian circle of the Kiev (University) Observatory were re-reduced in the FK 4 system. Two catalogues were published (N. A. Tchernega, Vest. Kievskogo Univ., ser. astronomii, no. 10, 1968 and no. 12, in press).

Reductions for FKSZ star declinations taking also into account the relations between the "Instrumental System and Observer" and "Fundamental System" deduced from the "Küstner Series" were finished at the La Plata observatory.

7500 positions of selected minor planets and the data for reference stars have been received at the Institute for Theoretical Astronomy (Leningrad) from 18 observatories of different countries (from 1949).

Thus the programme of observations of selected minor planets is nearing completion.

For determining the $\Delta \mathrm{A}$ and $\Delta \delta$ it is necessary to reduce the received Yale positions to a fundamental system, which evidently will be possible when the AGK3 and Southern photographical catalogues referred to the SRS positions are available.

At the Pulkovo Observatory determinations of absolute proper motions of stars relative to 250 galaxies in 85 areas continued. The preliminary results were reported at the 13th IAU General Assembly in Prague. An additional investigation was made of the magnitude equation. After taking the corrections into account a change was found in the previously given systematic corrections of the proper motions of AGK3R stars; in particular there is a considerable decrease in $\left(\Delta \mu_{\delta}\right)_{\delta}$.

A meniscus astrometric astrograph with a field of 25 sq. deg. was mounted on Mount El Roble (near Santiago, Chile) by the common efforts of Chilean and Soviet astronomers. A start was made on photographing the first epoch plates of 160 areas with galaxies in the declination zone $-90^{\circ}$ to $0^{\circ}$. 
At the Astronomical Institute of the Academy of Sciences of Uzbekistan (Tashkent Astronomical Observatory) the determination of absolute proper motions of stars relative to galaxies was continued according to the Pulkovo plan (Prof. Deutsch's plan). Plates taken more than 20 years ago with the Tashkent normal astrograph were used.

At the Sternberg Astronomical Institute absolute proper motions of 670 stars relative to the galaxies were determined. Plates taken with the 15-inch astrograph were used. Photographing of the first epoch plates of the regions containing selected FKSZ stars with the 15-inch astrograph of the Sternberg Institute has been completed.

The photographic observations (first epoch) according to the Pulkovo programme (galaxies) has been accomplished at the Golosseyevo observatory.

The astrographic observations of the galaxies (first epoch) with the Carte du Ciel Astrograph in the declination zone $-5^{\circ}$ to $-25^{\circ}$ of the Pulkovo programme has also been completed at the SanFernando (Cadiz) Observatory. A catalogue will be published in the near future. Photographic observations in the zone $-3^{\circ}$ to $-9^{\circ}$ for a second San Fernando catalogue are scheduled to begin in 1970.

\section{F. PZT and latitude star programmes}

The Askania Transit Circle of the Belgrade Observatory is being used for differential observations in the FK 4 system of 3941 stars from the zenith-telescope programmes. More than eleven thousand declination observations have been made. The observational programme will be finished in 1970 . The division errors were investigated using three methods (Heufelink, Bruns and a new method worked out by L. Nikolic - a collaborator of the Belgrade Observatory).

At the Sternberg Institute (Moscow) the zenith-telescope ZTL-180 is used for observations to obtain differential declinations of stars from all latitude programmes. The method used consists of micrometric measurements in narrow zones of 1:30. The reference catalogues used are FK 4 and AGK3R.

Observations in the FK 4 system ( $\delta$ only) with the new meridian circle of the Sternberg Institute of latitude and PZT stars, and observations in R. A. of the stars from the PZT programmes are being continued.

At the Odessa Observatory a catalogue of declinations of 194 stars from the Pulkovo ZTL-180 programme was compiled. The catalogue will be published in Trudy glavn. astr. Obs. Pulkovo.

A catalogue of declinations of the 192 stars from the new Moscow programme for the determination of the micrometer scale (wide pairs) was compiled at the same Observatory. The catalogue is in the press.

Corrections of the proper motions of the Washington zenith catalogue of scalar star pairs were determined at the Milan Observatory (Proverbio, Contr. Oss. astr. Milano-Merate, no. 317). At the Uccle Observatory the declinations and proper motions of all the stars from the ILS programme were deduced from discussions of 11501 positions from 463 star catalogues (P. Melchior et R. Dejaiffe, Ann. Obs. r. Belgique, 3rd series, vol. X, no. 3, 1969).

The Askania transit circle at Uccle was equipped with a device for photographing micrometer readings. The observations of 1102 stars (all latitude stars included) were begun at the end of 1968 .

Proper motions for 22 stars in the Herstmonceux-Calgary zenith zone were provided for PZT work at the Royal Greenwich Observatory.

Observations of 3941 stars in the latitude and PZT programmes have been continued with the vertical circle at Pulkovo. R.A. of PZT stars were observed with the meridian circle.

At the Engelhardt Observatory observations of these stars have been finished in the "clamp west" position of the meridian circle. Observations in the other position of the instrument have been begun.

\section{G. Astrolabe programme}

A general card-index of the star positions deduced from astrolabe observations made at different 
places was compiled at the Paris Observatory as preliminary work for compiling a general catalogue of stars observed with astrolabes.

The astrolabe observations made at Paris in 1956-69 have been studied preliminary with a view to use them for proper-motion determinations.

Observations made with the astrolabe in Haute-Provence for the improvement of FK 4 positions have been finished in December 1968. They are under dicussion.

Observations of the planets with astrolabes of some observatories have been organized by $\mathrm{Dr}$ Débarbat. She worked out a strict method for correcting these observations for the phase effect. (Bull. astr. ser. 3, 2, no. 4, 569, 1967). Results of the Moon observations received from instruments permitting observations at constant altitudes (astrolabes; circumzenithals) made it clear that such observations will be very useful and must be organized. (Bull. Soc. r. Sci. Liège, 38, No. 5-6, 205, 1969.)

Dr T. Okuda reports:

"At Mizusawa observations with the Danjon astrolabe have been made regularly since January 1966. The results of the observations have been published annually in the Bulletins, Time Service of Mizusawa Observatory. Provisional individual corrections $\Delta \alpha$ to 117 FK 4 and 24 FK 4 Sup stars and $\Delta \delta$ to 72 FK 4 and 7 FK 4 Sup stars were computed, all of which are included in the declination zone between $+9^{\circ}$ and $+69^{\circ}$. The mean errors $\varepsilon_{\Delta x}$ and $\varepsilon_{\Delta \delta}$ are comparatively large, and affected by those of group corrections" (Publ. int. Latit. Obs. Mizusawa, 6, no. 2, 185).

According to Dr D. V. Thomas more than 36000 transits were observed at the Cape Observatory with the Danjon astrolabe transferred from Herstmonceux. The observations were obtained during four years of operation of this programme, which was completed in April, 1969. A detailed analysis of the observations has now begun. Improved positions should eventually result for about 1000 stars brighter than 5.6 in the declination zone $-4^{\circ}$ to $-64^{\circ}$.

It has been shown that absolute declinations may be obtained in principle from observations made with a series of Danjon astrolabes suitably distributed in latitude or with a single reflecting instrument observing at two alternative altitudes (Herstmonceux Conference, 1969, Mon. Notes astr. Soc. Sth. Afr. 26, 2).

Dr L. Quijano reports from San Fernando (Cadiz):

"In April 1968 observations of 11 fundamental groups of stars were started mainly for the study of time and latitude. A selection of 250 FK 4 and FK 4 Supplement stars was made from these groups, 58 of which are observed in both E and W transits. From April 1969 on, small groups of catalogue stars were included in the observations. A rate of some 200 observations of fundamental groups per year is foreseen in which about 3000 transits can be inserted. The reductions are made in the Paris Observatory."

25000 transits have been observed at Pulkovo with the Danjon astrolabe during the last 7 years. Six annual cycles of observations have been discussed. Provisional corrections $\Delta \alpha$ and $\Delta \delta$ of about 300 stars in the declination zone $+30^{\circ}$ to $+80^{\circ}$ were thus obtained.

At Kriukovo (near Moscow), in Irkutsk and in Novosibirsk observations for time determinations were made. At Poltava the observations were produced for latitude determinations. All these observations may be used for the determination of star coordinate corrections.

\section{OBSERVATORIES AND INSTITUTES PROGRAMMES AND INVESTIGATIONS}

\section{Improvement of the Fundamental Catalogue and revision of the Albany General Catalogue}

Prof. W. Fricke reports from the Astronomisches Rechen-Institut at Heidelberg:

The work reported here is part of a current project of the Astronomisches Rechen-Institut, Heidelberg, to prepare future improvements of FK 4 and to revise the Albany General Catalogue.

Under the supervision of Lederle the documentation of star catalogues on punched cards has gone on steadily; these data are made available, according to the conception as started by IAU Working 
group on Space Ephemerides. Nowacki and Strobel (Heidelberg, 1968) have published the systematic differences between 71 observational catalogues and FK 3; these data formed part of the work on the construction of FK 4. By means of these systematic differences and the differences FK 4-FK 3 published in FK 4 any user may reduce the observational catalogues to the system of FK 4.

Work on new methods for deriving systematic differences between star catalogues was continued by Brosche. The differences are expanded in spherical harmonics, and all significant terms of the series development are taken into account. The number of terms in the series development is limited by the choice of $m=12$ and $n=22$ as the highest suffixes of the spherical harmonics. By means of the covariance matrix of the coefficients the mean error of the systematic differences is determined as a function of the position in the sky. The programme compiled for machine computation of systematic differences between FK 4 and important observational catalogues has the following advantages: optimal separation of systematic and individual parts of differences; consideration of the two-dimensional character of differences; the spectrum of coefficients forms a convenient basis for investigating sources of errors.

The determination of the correction to Newcomb's lunisolar precession has been the object of further investigations by Fricke: A redetermination on the basis of Newcomb's data has shown that Newcomb's error mainly originated from Auwers' declination system of proper motions of Bradley's stars; a rediscussion of equinox determinations has sufficiently cleared up the origin of the error in the motion of Newcomb's equinox; there is no variation of the obliquity of the ecliptic significantly different from Newcomb's value."

At the Pulkovo time service department a general catalogue of right ascensions of 807 stars to the 7 th magnitude in the declination zone $-30^{\circ}$ to $+90^{\circ}$ was compiled by N. N. Pavlyv, P. M. Afanacieva and S. S. Staritsyn. The catalogue is based upon 185371 observations, made during the IGY and IYC by the 9 Soviet time services. The mean epoch of the catalogue is close to 1958.0 . The meanerrors of the catalogue positions are: $\pm 0^{\circ} 0025\left(-10^{\circ}<\delta<+18^{\circ}\right), \pm 0.0022\left(20^{\circ}<\delta<40^{\circ}\right)$ and $\pm 0.037\left(55^{\circ}<\delta<76^{\circ}\right)$. The proper motions in $\alpha$ of 807 stars were obtained from a comparison of their right ascensions as given in the compiled general catalogue and those in FK 4, FK 3GC and N 30. Systematically these proper motions are completely independent from those of the fundamental systems.

A catalogue of $\alpha$ and $\delta$ in the FK 4 system of 972 stars in the visual magnitude range from 3.0 to 11.0 and in the declination interval from $-8^{\circ}$ to $+65^{\circ}$ based upon observations made at the Brørfelde (Copenhagen University) Observatory from August 1964 to January 1967 was published (Publ. mind. Medd. Kbh. Obs., 197, 1968). The catalogue includes stars from 6 lists: FK 4 Supplement stars; $\mathrm{O}$ and $\mathrm{B}$ types stars nearer than 20 parsec, high velocity stars, brighter $\mathrm{G}$ and $\mathrm{K}$ stars in the photometric classification programme in progress at the Brørfelde and Lowell observatories, and variable and double stars selected by G. van Herk.

The automated photographic micrometer described by Lausten in 1967 was used. Most stars were observed four times, but all stars in the programme which were observed at least two times in right ascension and declination were included in the catalogue. The following mean errors of a single observation in right ascension and declination were found:

$$
\varepsilon_{\alpha} \cos \delta= \pm 0.0152 \pm 0.0002 ; \quad \varepsilon_{\delta}= \pm 0.219 \pm 0.003
$$

At the Golosseyevo Observatory (near Kiev) a catalogue of absolute declinations of 1181 bright (FK 3) stars in the zone $-31^{\circ}$ to $+90^{\circ}$ and 611 faint (FKSZ) stars in the zone $-26^{\circ}$ to $+90^{\circ}$ has been published (A. K. Korol, Declinations of Bright and Faint Stars in a Common System, Naukova Dumka, Kiev, 1969 (in Russian)). The catalogue is based on the observations (more than 12000) made with the Wanschaff vertical circle (D $19 \mathrm{~cm}, \mathrm{~F} 252 \mathrm{~cm}$ ) in 1954-62. The instrument was carefully investigated, especially its flexure. The observations of stars were reduced with the values of flexure determined during the same nights. The mean error of one observation is represented by the formula

$$
\delta(z)^{2}=(0.320)^{2}+(0.165 \operatorname{tg} z)^{2}
$$


The catalogue is close to the fundamental systems FK 4 and N 30 (a little nearer to the latter).

The results of observations made with the six-inch transit circle of the U.S. Naval Observatory between 1956-62 have been published (Adams, A. N. and Scott, D. K., U.S. Naval Observatory Publ., 19, part 2, 1968). The six-inch transit circle observations of the Moon made in Washington in 1925-68 have been reduced with the revised system of astronomical constants adopted by the IAU in 1964 and adjusted to the FK 4 system. The observed positions were compared with the Improved Lunar Ephemeris. The results are published (U.S. Naval Obs. Publ., 19 part 3). An analysis of these results by B. L. Klock and D. K. Scott for equator and equinox corrections and for corrections to the lunar orbital elements is published in the Astronomical Journal.

The current six-inch transit circle USNO programme of fundamental observations of the Sun, Moon, planets, four bright asteroids, FK 4 and FK 4 Supplement stars and "Bright Stars" north of $-30^{\circ}$ declination will be completed in 1970 .

A catalogue of R.A., Declination and proper motions of 520 circumpolar stars in the declination zone from $+84^{\circ}$ to $90^{\circ}$ was published by Telniuk-Adamchuk (Astr. Obs. Kievsk. Univ., Kiev, 1969). The positions of stars are based on the observations made in 1876-82 by Fabritius with the Repsold meridian circle of the Kiev Univ. Obs.; the proper motions were determined using 9 catalogues of circumpolar stars from the catalogue of Carrington up to AGK3.

At the Sternberg Astron. Inst. (Moscow) a general catalogue of R. A. of circumpolar stars is being compiled by A. P. Guliaev. Observations made at several Soviet observatories in previous years have been used.

Reductions of the observations of circumpolar stars made in 1909-14 by L. O. Struve. N. N. Evdokimov and B. Y. Kudrevich with the meridian circle of the Kharkov Observatory are in progress. The first part of the work - the compilation of a catalogue of declinations of 1047 circumpolar stars - will be accomplished in the near future. Reductions of the semi-absolute determinations of the declinations of 270 stars made in 1924-33 by N. N. Evdokimov and B. P. OstachenkoKudriavcev are also in progress.

The first Greenwich catalogue for 1950, based on observations made with the Airy Transit Circle 1931-38 has been published (Woolley et al., Roy. Greenwich Obs. Annals, No. 3, 1969). The contents of the catalogue were made available many years before publication and have been incorporated in the N 30 and FK 4 catalogues.

At Herstmonceux the programme of observing stars with good radial velocities and poor proper motions has been concluded. Lists of selected variable stars have been observed in various differential and fundamental programmes. Groups of reference stars in the vicinity of quasi-stellar sources were observed differentially with groups of FK 4 stars in order to provide a systematic framework for photographic positions of faint optical objects identified with the sources.

Proper motions for 182 semi-regular and RV Tauri variables have been published with an account of the methods employed (Roy. Obs. Bull., 142). Proper motions of 19 Praesepe stars have also been determined.

Observations of absolute declinations of FK 4 stars, major planets, and of the four brightest minor planets are being continued with the vertical circle of the Munich Observatory in Munich.

The compilation of a catalogue of positions ( $\delta$ and $\alpha$ ) and proper motions of 130 eclipsing variable stars based on the observations with the meridian circle at the Odessa Observatory is almost completed. The catalogue will be published in 1970 .

Observations of 9537 zodiacal stars up to the visual magnitude 8.0 (in $\beta$ limits from $-15^{\circ}$ to $+15^{\circ}$ ) were started by I. E. Gordon with the Repsold meridian circle of the Nikolaev Observatory. It would be of great importance that other observatories join this work.

An investigation of the systematic errors of some AG catalogues in equatorial zones was made by L. F. Gorel using the results of observations of AGK3R and KSZ stars made at the Nikolaev Observatory in previous years. She has compiled a catalogue of proper motions of 12590 AGK3R and KSZ stars and determined the correction to the precessional constant.

At the Besançon Observatory the meridian observations of the stars, the occultations of which had been observed at the observatory, were started. In the computing department of this obser- 
vatory the proper motions of the 3rd catalogue of Besançon (List of Blaauw stars) were prepared for publication. The differential positions of the fundamental stars from the 2nd catalogue of Besançon were also prepared for print.

Regular observations of the Sun and major planets (especially Mars and Venus) and fundamental stars were continued also at many observatories (Bickley, Herstmonceux, Kazan (Engelhardt) Kharkov, Kiev (University), Milan, Munich, Moscow (Sternberg Institute), Nikolaev, Pulkovo, Tashkent, Tokyo and Washington). At some observatories the four brightest minor planets were observed too (Bickley, Herstmonceux, Milan, Munich, Tokyo and Washington).

I was also informed that photographic observations of the major and bright minor planets were made at Nikolaev, Golosseyevo, Moscow-Sternberg Institute and at Pulkovo.

The results of observations of bodies of the Solar System are partly published (Vestnik Kievsk. Univ. Ser. Astronom., nos. 11 and 12, 1969 (in Ukrainian with a Russian résumé); Circ. astr. Obs. Milan, nos. 23 and 25, 1967; Ann. Tokyo astr. Obs. 5, 1967; Trudy Pulkovo Obs., 77, 1969, Izv. glavn. astr. Obs. Pulkovo, no. 183, 1968; Bjull. Inst. teor. astr. (Leningrad) 5 (128)).

The Second Cape Catalogue for 1950.0 including all Sun, Moon and Planet observations made at the Cape between 1935 and 1960 was published in 1969.

A discussion of the absolute observations in $\delta$ made at the Melbourne Observatory in 1928-41 has been completed at Pulkovo. The corresponding catalogues of absolute declinations of 2466 stars have been published: (K. N. Tavastsherna, The catalogue of Declinations of 2249 BacklundHough List Stars from +35 to $-90^{\circ}$ of Declination, observed at the Melbourne observatory during 1928-41. $\mathrm{Me}_{s 0} \mathrm{I}(\delta)$, and K. N. Tavastsherna and N. A. Schacht - three small declination catalogues $\mathrm{Me}_{50} \mathrm{II}(\delta) \mathrm{Me}_{50} \mathrm{III}(\delta)$ and $\mathrm{Me}_{50} \mathrm{IV}(\delta)$. Edition of the Pulkovo Observatory, Leningrad, 1968).

The discussion of the corresponding observations in right ascension is continued.

Observations of absolute declinations of the Southern Hemisphere stars of the FK4 catalogue are being continued at the Southern Astrometric Station in La Leona.

Dr M. S. Zverev reports: "In connexion with the observations of SRS, BS, DS and FK 4 stars with the meridian circle of the Cerro Calan Observatory (Chile) corrections $\Delta \alpha_{\delta}$ for 1044 southern FK 4 stars were determined, using a 'quasi absolute method' (M. S. Zverev, Russ. astr. J. 46, 6, 1969) on the base of about seven thousand observations (211 fundamental series). It was shown that the FK 4 system needed corrections of the form $\Delta \alpha_{b}$ in the equatorial region and especially in the declination zone close to $-35^{\circ}$ and $-75^{\circ}$ (C. Anguita, G. Carrasoo, D. D. Polojentsev, M. S. Zverev, Astr. Cirk. U.S.S.R. 506, 1969; Inf. Bull. Southern Hemisphere, 15, 1969).

Large systematic errors of the Cape catalogue of circumpolar stars (W. Gliese, R. Obs. Bull. 106, London, 1966) in the Declination zone $-80^{\circ}$ to $-83^{\circ}$ have been revealed.

All these results were confirmed on the basis of observations of 839 bright southern stars made at Cerro Calan observatory with the Zeiss transit by P. R. Loyola and V. N. Shishkina (Publ. no. 4, Obs. Cerro Calan, Santiago de Chile, 1968, V. N. Shishkina, M. S. Zverev, Inf. Bull. Southern Hemisphere, 16, 1970, in print).

Dr von der Heide reports from the Perth Observatory on the determination of $\Delta \alpha_{\delta}$ and $\Delta \delta_{\delta}$ corrections from differential observations of the SRS and BS stars. He remarks that "the $\Delta \alpha_{\delta} \cos \delta$ seems to have a maximum of -0.35 at $\delta=-65^{\circ}$; the $\Delta \delta_{\delta}$ go up to about -0.5 at $\delta=-35^{\circ}$."

S. Slaucitajs reports from the La Plata Observatory. "The possibility is being considered for the determination by means of 'Küstner Series' of systematic errors $\Delta \alpha_{a}$ and $\Delta \alpha_{\mathfrak{d}}$ that have been observed in previous years as well as those that are still in observation."

At the Yale Observatory in collaboration with the Numerical Analysis Group, Section 312 (U.S.A.) the final solution for the Yale Minor Planets Project was obtained. 6912 observations of 15 minor planets were used. Corrections to the six orbital elements for each planet and 108 corrections of $\Delta \alpha_{\kappa}$ and $\Delta \delta_{\kappa}$ for 54 regions in the declination zone from $-30^{\circ}$ to $+30^{\circ}$ for the Yale Zone Catalog and 120 corrections for 60 regions for GC have been obtained (198 (210) corrections in total).

To solve the system of 6812 equations with 198 or with 210 unknowns (for GC), computer 
programmes and techniques developed by the Numerical Analysis Group for the IBM 7040-7094 and modified for use on the UNIVAC 1108 were used. This solution corrects the internal inconsistencies within the system of right ascensions and declinations and in addition it adjusts the location of the equator $\left(\Delta \delta_{0}=+0\right.$.17). It makes no adjustment to the location of the equinox along the equator $-\Delta A$. The results will be published in the Astronomical Papers of the American Ephemeris in 1970.

H. Yasuda (Tokyo Observatory) has found the periodic errors $\Delta \alpha_{\alpha}$ and $\Delta \delta_{\alpha}$ of the FK 4 system in the equatorial zone from meridian observations of the four brightest minor planets (Ann. Tokyo astr. Obs., 5, 1967). K. Hurukawa showed the same trend of $\Delta \delta_{\alpha}$ in the declination zone of $+30^{\circ}$ from floating zenith tube observations at the Mizusawa Latitude Observatory and found a reliable coincidence among three independent determinations, the determination from minor planets, with a floating zenith tube and with the astrolabe at the Paris Observatory (Pub. int. Latit. Obs. Mizusawa, 6, 1968).

Dr G. van Herk reports from the Leiden Observatory:

"I have continued my investigations related to possible sources of systematic errors in meridian circle catalogues from

Besançon, Ann. Nouv. Série, 6, 1964.

Brørfelde, Publ. Københavns Obs., no. 197, 1968.

München, Veröff., 5, no. 15, 1963.

Paris, J. Obs., 42, 94, 1959.

Washington, Publ. U.S.N.O., 19, part 1, 1964.

It turns out that the right ascensions agree better amongst each other than the declinations, in all respects. The declinations for Washington show some dependency on spectral type, derived from the published differences between upper and lower culmination, which is understood according to the lines of $B A N, 11,489,494,1952$. The differences in right ascension of various catalogues show in some cases some dependency on magnitude, that is the nominal magnitudes. As long as the true, observed, magnitudes are not published, this parameter is only inadequately known. The $\Delta \alpha$ for Washington and FK 4 show also a dependency on magnitude. I refer to $B A N, 19,355,1968$."

"A careful discussion of vertical circle observations made at Pulkovo, Odessa, Munich and Canberra has proved the existence of an annual variation of the constant of refraction with an amplitude of about 0."1" writes Dr F. Schmeidler from the Munich observatory.

From the Goethe Link Observatory (Indiana University) Dr R. d'E. Atkinson writes: "I am engaged on a re-evaluation of the results obtained with the Cookson Floating Zenith Telescope, 1911-36, to study the possibility (suggested by Jeffreys) that the leading terms in the nutations in longitudes and in obliquity may not be related in the manner required by a rigid earth. $I$ hope to complete this work before the General Assembly."

New computing techniques have been used at many observatories for the reduction of observations. At some observatories new methods of reduction have been discussed. At the Golosseyevo Observatory the theory of the accidental sequences and fields has been used to study the distribution of the errors of observations with the Wanschaff vertical circle (Intern. Lat. star programmes). It was shown that the empirical distribution departs substantially from the normal one. Thus it is recommended to use weights in accordance with S. Newcomb's and G. Jeffreys' method.

D. P. Duma at this observatory has discussed problems connected with the determination of equator and equinox corrections from observations of major and minor planets and artificial satellites.

At the Sternberg Astron. Inst. (Moscow) a strict method for reductions of the differential observations to the fundamental system was worked out (L. M. Chommik).

A. P. Gulaiev from the same Institute has proved that it is necessary to investigate the systematical errors of the observations of the programme stars before using them for determinations of the parameters of the meridian circle.

A project for the determination of more definitive proper motions of the early type and Cepheid stars of Blaauw's list is being carried out under the supervision of R. W. Rhynsburger (USNO). 
At the same observatory the project for placing star catalogues on punched cards has been continued by the Nautical Almanac Office.

Improved reconstructions and investigations of the meridian instruments and determinations of the instrumental and personal errors have been made at many observatories.

Information on work of this kind has been received from the observatories: Belgrade, Besançon, Brussels, Bucharest, Golosseyevo, Greenwich-Herstmonceux, Kharkov, Kazan-Engelhardt, Lund, Milan, Moscow-Sternberg Inst., Nikolaev-Odessa, Potsdam, Pulkovo.

Some work of this kind was already mentioned in previous parts of this report.

I would like only to give some details on the investigation of the division errors of the circles manufactured by Askania and Repsold made by P. Melchior using spectral analysis. The results will be published in the near future. The same method was used by K. G. Gnevysheva (Pulkovo) for investigation of some Askania circles.

A detailed investigation of the flexure of the Wanschaff vertical circle (Golosseyevo) was made by A. S. Kharin. It was shown that the flexure of this instrument is correlated with the temperature gradients (not with the temperature itself). The same method was used for the investigation of flexure at the vertical circle of the Belgrade Observatory (A. S. Kharin, G. K. Teleki).

A. I. Nefed'eva (Kazan-Engelhardt Observatory) has shown that systematical errors of the zenith distances measured with the meridian circle of this observatory remained unchanged during 120 years.

In the Vsesousny Nauchno-Issledovatelsky Institut Fisiko-technich. i Radiotechnich. Izmerenij (VNIIFRTRI (Moscow)) B. I. Vlasov proposed a method to improve fundamental catalogues by using space observations. The results were reported at the 18 th Astrometrical Conference of the U.S.S.R. (Pulkovo 31 May-5 June 1969) and will be published in the Trudy of this Conference.

The second edition of the book by V. V. Podobed, Fundamentalnaja Astrometria (Fundamental Astrometry) was published in 1968.

\section{SUGGESTED TOPICS FOR DISCUSSIONS AT THE GENERAL ASSEMBLY}

1. Recent progress in instruments and their accessories.

2. Progress with the existing mirror transit circles.

3. Results obtained with new types of meridian instruments.

4. The organization of absolute observations of stars at as many observatories as possible.

5. The international collaboration for the automation of reductions of observations.

6. The correction of the FK 4 for the southern hemisphere on the basis of modern meridian observations.

7. The reduction of differential observations to a fundamental system.

8. The discussion of the results of the SRS observations.

9. Theory of astronomical refraction based on new meteorological and aerological data.

10. The problem of site-testing for astrometry with Danjon astrolabes.

11. The refraction in pavilions.

12. The possibility to organize Symposia in 1971-72 on these problems: Cosmical and lunar astrometry; applicability of radio and laser astronomy to position astronomy; the project to refer motions to the system of the galaxies; the use of observations of bright minor planets in fundamental astronomy; distribution of data in machine-readable form and bringing existing data (e.g. large catalogues of positions and proper motions, $\mathrm{BD}$ or $\mathrm{HD}$ included) into machine-readable form by reasonable international cooperation.

13. The name of our commission: Dr Dieckvoss proposes "Basic Astrometry of Position". 